\title{
TAVI: thoughtful application of valve treatment innovations
}

\author{
Nikos Werner · Georg Nickenig
}

Published online: 6 February 2014

(c) Springer-Verlag Berlin Heidelberg 2014

Keywords TAVI - TAVR · PARTNER - SURTAVI ·

Registry · Patient selection - Valve types · Valve in valve

\section{Introduction}

Transcatheter aortic valve implantation (TAVI) is the standard of care in inoperable, symptomatic patients with severe aortic stenosis (AS) and an appropriate life expectancy. It has emerged as an acceptable alternative in patients with AS and high risk for surgical aortic valve replacement (SAVR). These insights derive from the randomized PARTNER studies that compared for the first time conservative treatment versus TAVI and TAVI versus SAVR [1, 2]. Just recently, we learned from the US Pivotal Trial (presented at TCT conference 2013) that in high-risk patients (estimated mortality or irreversible morbidity $\geq 50 \%$ ), TAVI is superior in terms of mortality and morbidity compared to an objective performance goal [3].

While these two studies comprise 1,500 patients, more than 100,000 procedures have already been performed worldwide. Many of these patients have been included into observational registries organized by independent researchers, professional societies, companies, and government agencies. The vast majority of insights currently used in clinical practice, when performing TAVI procedures, are derived from these registry data. A number of large-scale TAVI registries, e.g., the FRANCE-2 registry [4], the German Aortic Valve Registry (GARY) [5], and

N. Werner $(\bowtie) \cdot$ G. Nickenig

Medizinische Klinik und Poliklinik II, Universitätsklinikum

Bonn, Rheinische Friedrich-Wilhelms-Universität Bonn,

Sigmund-Freud-Str. 25, 53105 Bonn, Germany

e-mail: nikos.werner@ukb.uni-bonn.de the US TVT Registry [6], close some gaps between highly expensive and long-lasting randomized trials with limited and highly selected patients and the need for "real-world" data that are promptly available.

In this issue of CRCD, Haussig et al. give us an excellent overview of several TAVI registries and their possible clinical implications. Scientific value of all registries is of course limited, since consecutive enrollment of patients is usually not vigorously controlled and assured and can be as low as $50 \%$ per center. Also, follow-up rate for each and any end point is frequently not reported or not uniformly in a high and acceptable range. However, one of the most valuable insights of these registries is the continued decrease in morbidity and mortality over time. With more operator experience and better patient selection, the rate of irreversible morbidity, such as major stroke, has continuously decreased and is well below $3 \%$ after 30 days in contemporary registries. Realistic 30-day mortality rates are below $5 \%$ depending on the comorbidities of treated patients. Again, all data available describe a clear correlation between pre-operative comorbidities and post-procedural outcome with a stepwise decrease in mortality rates with lower rates of TAVI-complicating morbidities [7]. In a recent analysis, Makkar and colleagues further address this topic; patients in the PARTNER B cohort (inoperable patients) were classified based on whether or not they were technically inoperable or clinically inoperable. While technically inoperable patients suffered mainly from porcelain aorta, reasons to decline patients for surgery from a clinical point of view included frailty and systemic comorbidities. Two major conclusions result from this analysis: (1) irrespective of the reason for inoperability, both groups had a significant survival benefit compared to standard conservative treatment; (2) mortality in technically inoperable patients was significantly lower at 2 years 
compared to clinically inoperable patients (23.3 vs. $43.8 \%, p<0.001)$, highlighting the importance of morbidity-based patient selection [8].

Obviously, 1 year mortality is still unacceptably high for such an elaborated procedure warranting further efforts in patient selection and procedural performance. O'Sullivan and colleagues address well the topic of patient selection in this CRCD issue, discussing clinical risk scores and anatomical features influencing decision making in TAVI. Multiple scores try to estimate perioperative 30-day mortality (STS, logistic EuroScore, German Aortic Valve Score) based on clinical characteristics, but we still lack a comprehensive score including clinical and anatomical features allowing discrimination between the futile and the optimal patient. One has to keep in mind that most scores have been developed from the surgical perspective and that a reliable TAVI risk score is still to be developed.

While this discussion is ongoing and clear-cut decisionmaking algorithms are lacking, decision making in patients with degenerated surgical aortic valves and considerable risk for a redo procedure appears to become easier.

Valve-in-valve (ViV) implantation using a TAVI prosthesis is the first alternative treatment option for these patients who had to undergo redo surgery at an increased risk in recent years. The operative mortality for an elective redo aortic valve surgery is reported to range from 5 to $11.5 \%$ with up to $30 \%$ in high-risk or emergency patients. Mortality rates of redo surgery for failing mitral valve prosthesis are even higher (up to $15 \%$ ). As reviewed by Milburn et al., ViV procedures are an optimal alternative in patients with failing aortic valves (both, in restenosis and aortic regurgitation), but may be extended to mitral valve, pulmonary, and tricuspid valves. An explosion of case reports describing $\mathrm{ViV}$ procedures for all valves has been noted in recent years. From these data and the recently published worldwide registry for $\mathrm{ViV}$ procedures, we have learned that exact knowledge about the implanted surgical valve is of pivotal importance in order to perform a successful ViV procedure. Milburn and colleagues describe in detail and step by step the approach and technical aspects of the ViV procedure and pay close attention to the avoidance of typical complications such as inaccurate valve sizing, malpositioning, and coronary obstruction. Interestingly, when discussing valve replacement strategies with heart surgeons, a clear preference toward biological valves compared to mechanical valves is noted in recent years, with the option of having $\mathrm{ViV}$ procedures available in case of prosthesis failure at 10-15 years after surgical valve replacement. Currently, we clearly lack long-term results and durability data on ViV procedures compared to redo surgery. However, when current experience with ViV procedures further expands, larger registry data become available, and dedicated valves for $\mathrm{ViV}$ procedures are developed, the future of $\mathrm{ViV}$ procedures appears very bright.

\section{What else do we need to bring TAVI to an even brighter future?}

Refinements are needed in pre-, peri-, and post-procedural patient's care.

Patient selection is far from perfect and better risk stratification tools are needed. During and shortly after valve implantations, pace maker rate, aortic regurgitation, and vascular access site complications are vexing problems. Predictability of the implantation process is still unacceptably low. Most of these issues are being addressed by innovative device design. New devices have a lower entry profile going down to $14 \mathrm{~F}$ inner diameter and reducing vascular complications. However, closure device success is still unpredictable, especially in severely calcified atherosclerotic access sites. Novel closure devices are under development, but have not entered the market, yet.

We currently witness a continuous development of novel valve systems. Up to date, five valves for the transfemoral route have been CE certified (Edwards Sapien, Medtronic CoreValve, Direct Flow Medical System, St. Jude Portico, and Boston Lotus Valve) and four additional for the transapical route (Edwards Sapien, Medtronic Engager, Symetis Acurate, JenaValve System). Repositionability is a common characteristic of most of the new valves that will help to increase implantation quality (and lower aortic regurgitation and pace maker rate), especially in difficult anatomies. Maybe, these are the first steps toward an anatomy-adapted valve selection process. However, we need to keep in mind that novel devices call for novel experience and unexpected problems and complications. With an increasing number of valves on the market, selection algorithms appear necessary to treat the individual patient with the perfectly fitting valve.

Post-procedural treatment regimens currently lack evidence. What is the right dose and length of platelet inhibition? Should we consider even anticoagulation in this population prone to atrial fibrillation? What is the correct heart failure medication after resolved valvular cardiomyopathy, and do we need a special emphasis on hypertrophy regression? At the moment, we are far away from answering these questions in a scientific way.

Finally, we can observe a worldwide trend toward TAVI treatment in lower-risk patients. Clearly, randomized data in intermediate-risk patients are urgently needed. In this respect, registry data with selection bias, non-monitored data, and lack of clear comparative measurements are hypothesis generating, but not sufficient to argue in favor or against TAVI in intermediate-risk patients. Hopefully, at 
latest in 3-5 years, we will get another review series at this point about the results of the ongoing randomized trials PARTNER II and SURTAVI that evaluate intermediaterisk patients.

Only 11 years after the first TAVI procedure, transcatheter valve implantation in inoperable and high-risk patients with native aortic stenosis or degenerated surgical valves has emerged as a safe and effective procedure bringing high-quality life back to most of our patients. This high-speed development of innovations needs watchful guidance and proper scientific evaluations. If so, chances are great that we and our colleagues will perceive this venue as another revolution in cardiovascular medicine in a few decades from now.

Conflict of interest Both authors received speakers' honorarium and research support from Medtronic and Edwards Lifesciences.

\section{References}

1. Leon MB, Smith CR, Mack M et al (2010) Transcatheter aorticvalve implantation for aortic stenosis in patients who cannot undergo surgery. N Engl J Med 363:1597-1607
2. Smith CR, Leon MB, Mack MJ et al (2011) Transcatheter versus surgical aortic-valve replacement in high-risk patients. N Engl J Med 364:2187-2198

3. Popma JJ (2013) on behalf of the Corevalve US clinical investigators. Corevalve US pivotal trial extreme risk iliofemoral study results. Presented at TCT, San Francisco, USA, 28 Nov 2013

4. Gilard M, Eltchaninoff H, Iung B et al (2012) Registry of transcatheter aortic-valve implantation in high-risk patients. N Engl J Med 366:1705-1715

5. Hamm CW, Möllmann H, Holzhey D et al (2013) The German Aortic Valve Registry (GARY): in-hospital outcome. Eur Heart J [Epub ahead of print]

6. Mack MJ, Brennan JM, Brindis R et al (2013) Outcomes following transcatheter aortic valve replacement in the United States. JAMA 310:2069-2077

7. Wenaweser P, Stortecky S, Schwander S et al (2013) Clinical outcomes of patients with estimated low or intermediate surgical risk undergoing transcatheter aortic valve implantation. Eur Heart J 34:1894-1905

8. Raj R Makkar MD, Hasan Jilaihawi MD, Michael Mack MD et al. (2013) Stratification of Outcomes after Transcatheter Aortic Valve Replacement According to Surgical Inoperability for Technical Versus Clinical Reasons. J Am Coll Cardiol. doi:10.1016/j.jacc. 2013.08.1641 